Sharif University of Technology
Scientia Iranica
Transactions B: Mechanical Engineering
IRAN

\title{
Slip flow of nanofluid over a stretching vertical cylinder in the presence of non-linear thermal radiation and non-uniform heat source/sink
}

\author{
C.S. Sravanthi* \\ Department of Mathematics, The M.S. University of Baroda, Vadodara, Gujarat, India. \\ Received 24 July 2016; received in revised form 3 May 2017; accepted 18 September 2017
}

\author{
KEYWORDS \\ Nanofluid; \\ Stretching vertical \\ cylinder; \\ Non-linear thermal \\ radiation; \\ HAM.
}

\begin{abstract}
An analysis is performed to study axisymmetric mixed convective boundary layer flow of a nanofluid over a vertical stretching circular cylinder in the presence of nonlinear radiative heat flux. The effects of non-uniform heat source/sink and slip flow are also taken into consideration. Water as conventional base fluid containing nanoparticles of Copper $(\mathrm{Cu})$ is used. By means of similarity transformations, the governing partial differential equations are reduced to highly non-linear ordinary differential equations and then solved analytically using Homotopy Analysis Method (HAM). A comparison is made with the available results in the literature, which shows that our results are in very good agreement with the known results. A parametric study of the physical parameters is made and results are presented through graphs and tables. The results indicate that the thermal boundary layer is thicker for non-linear thermal radiation problem than for linear thermal radiation. It is also found out that heat transfer rate at the surface decreases with increase in both space- and time-dependent heat source/sink parameters.
\end{abstract}

(C) 2018 Sharif University of Technology. All rights reserved.

\section{Introduction}

Thermal conductivity rate of ordinary base fluids, including water, ethylene glycol, and oil, is very low. However, nowadays, cooling of electronic devices is a major industrial requirement. To achieve this, the nanoscale solid particles are submerged into host fluids, which change thermophysical characteristics of these fluids and dramatically enhance heat transfer rate. These fluids are said to be nanofluids. Choi [1] was the first who identified this colloidal suspension. The nanofluids have applications in cooling of electronics, heat exchanger, nuclear reactor safety, hyperthermia, biomedicine, engine cooling, vehicle thermal manage-

\footnotetext{
*. E-mail address: srinivasan_sravanthi@yahoo.com
}

doi: $10.24200 /$ sci.2017.4580 ment, and many others. Pop et al. [2] investigated the mixed convective boundary layer flow of a nanofluid over a vertical circular cylinder using homotopy analysis method. A bulk of research articles on nanofluids and cylinder stretching flows is available in the literature of which few can be seen in the [3-18].

Heat transfer, influenced by thermal radiation, has applications in many technological processes, including nuclear power plants, gas turbines, and various propulsion devices for aircraft, missiles, satellites, and space vehicles. Many investigations [19-22] into thermal radiation effect by applying the linearized form of Rosseland approximation, which is obtained by assuming sufficiently small temperature differences within the flow, have been carried out. However, linear radiation is not valid for high temperature difference and the dimensionless parameter used in the linearized Rosseland approximation is the only 
effective Prandtl number [23]. Recently, the nonlinear Rosseland diffusion approximation has been adopted by various researchers [24-33] for studying the nonlinear radiative heat transfer in various geometries. The nonlinear Rosseland diffusion approximation is valid for small as well as large temperature difference of surface and ambient fluid, unlike the linearized Rosseland approximation, which is valid only for sufficiently small temperature difference.

The study of heat source/sink effects on heat transfer is very important, because these effects are crucial in controlling the heat transfer. Hayat et al. [34] studied the non-uniform heat source on unsteady heat and mass transfer MHD flow of nanofluids over a stretching sheet. Manjulatha et al. [35] performed a thermal analysis by conducting dusty fluid flow in a porous medium over a stretching cylinder in the presence of non-uniform source/sink using Runge Kutta Fehlberg-45 Method. Subhas et al. [36] investigated the effect of non-uniform heat source on MHD heat transfer in a liquid film over an unsteady stretching sheet. Effect of non-uniform heat generation on unsteady MHD non-Darcian flow over a vertical stretching surface with variable properties was studied by Muthamilselvan et al. [37].

No-slip boundary condition (the assumption that a liquid adheres to a solid boundary) is used to obtain the solution to all the above-mentioned studies. The no-slip boundary condition is one of the central assumptions of the Navier-Stokes theory. However, partial velocity slip (the non-adherence of the fluid to a solid boundary) may occur on the stretching boundary when the fluid is particulate, such as emulsions, suspensions, foams, and polymer solutions. Recently, many researchers [38-44] have investigated the flow problems taking slip flow condition at the boundary into account. Polishing of artificial heart valves and internal cavities is an important technological application of the fluids that exhibit boundary slip.

For some of the above-mentioned problems, numerical techniques have been developed to obtain the accurate solution for years. But, due to some restrictions [45], scientists have considered analytical approaches as an alternative. One of the most common methods in this field, which is widely applied in science and engineering, is perturbation technique. It must be noted that perturbation technique cannot be applied to strongly nonlinear problems, as it strongly depends upon small/large physical parameters. In order to avoid the dependency on small/large parameters, nonperturbation techniques such as Adomian decomposition method and variational iteration method have been developed. The major disadvantage of these methods is that they cannot ensure the convergence of series solution. On the other hand, the Homotopy Analysis Method (HAM) proposed by Liao [46] is a general analytical approach to obtain series solutions to strongly nonlinear equations, which can provide us with a simple way to ensure the convergence of solution series. Recently, the HAM has been adopted by various researchers [47-50] to obtain solutions to various fluid flow problems.

No attempt has been made so far to analyze the boundary layer flow of nanofluid over a vertical stretching cylinder with non-linear thermal radiation and non-uniform heat source/sink under slip flow boundary conditions. The present work aims to fill the gap in the existing literature. In this article, we give a detailed discussion of the boundary layer slip flow and heat transfer of nanofluid over a stretching vertical cylinder in the presence of non-linear thermal radiation and non-uniform heat source/sink, analytically, via Homotopy Analysis Method (HAM). The effects of different emerging parameters are demonstrated through graphical representations and discussed at length.

\section{Formulation of the problem}

Let us consider the steady axisymmetric mixed convective boundary layer flow of a $\mathrm{Cu}$-water nanofluid over a vertical circular stretching cylinder with slip conditions. Flow analysis is carried out with non-linear thermal radiation and non-uniform heat source/sink. Cylindrical coordinates are chosen in such a way that the $x$-axis is along the axial direction of the cylinder while $r$-axis is normal to it (Figure 1). Stretching velocity of the cylinder is due to the application of two forces to the cylinder, which are equal in magnitude but opposite in direction, when origin is kept constant. Here, viscous dissipation is neglected. Under these assumptions and using boundary layer approximations, the conservation laws are given as follows [2,24,34]:

$$
\frac{\partial(r u)}{\partial x}+\frac{\partial(r w)}{\partial r}=0
$$

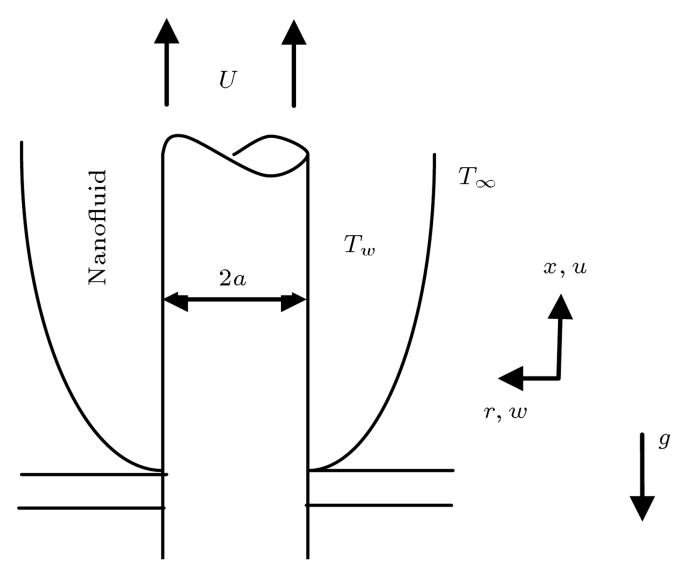

Figure 1. Physical model and coordinate system of the problem. 


$$
\begin{aligned}
& u \frac{\partial u}{\partial x}+w \frac{\partial u}{\partial r}=\frac{\nu_{n f}}{r} \frac{\partial}{\partial r}\left(r \frac{\partial u}{\partial r}\right) \\
&+\frac{\varphi \rho_{s} \beta_{s}+(1-\varphi) \rho_{f} \beta_{f}}{\rho_{n f}} g\left(T-T_{\infty}\right), \\
& u \frac{\partial T}{\partial x}+w \frac{\partial T}{\partial r}=\alpha_{n f} \frac{1}{r} \frac{\partial}{\partial r}\left(r \frac{\partial T}{\partial r}\right) \\
& \quad-\frac{1}{\left(\rho c_{p}\right)_{n f}} \frac{1}{r} \frac{\partial\left(r q_{r}\right)}{\partial r}+\frac{1}{\left(\rho c_{p}\right)_{n f}} q^{\prime \prime \prime}
\end{aligned}
$$

The corresponding boundary conditions are [38-40]:

$$
\begin{array}{ll}
u=U(x)+B_{1} \frac{\partial u}{\partial r}, & w=0, \\
T=T_{w}(x)+K_{1} \frac{\partial T}{\partial r} & \text { at } r=a, \\
u \rightarrow 0, \quad T \rightarrow T_{\infty} & \text { as } r \rightarrow \infty .
\end{array}
$$

Here, $\nu_{n f}$ is kinematic viscosity of the nanofluid, $\rho_{n f}$ is density of nanofluid, and $\alpha_{n f}$ is thermal diffusivity of the nanofluid, which are given by [3]:

$$
\begin{aligned}
& \nu_{n f}=\frac{\mu_{f}}{(1-\varphi)^{2.5}\left[(1-\varphi) \rho_{f}+\varphi \rho_{s}\right]}, \\
& \rho_{n f}=(1-\varphi) \rho_{f}+\varphi \rho_{s}, \\
& \alpha_{n f}=\frac{k_{n f}}{\left(\rho c_{p}\right)_{n f}}, \quad \mu_{n f}=\frac{\mu_{f}}{(1-\varphi)^{2.5}}, \\
& \left(\rho c_{p}\right)_{n f}=(1-\varphi)\left(\rho c_{p}\right)_{f}+\varphi\left(\rho c_{p}\right)_{s}, \\
& \frac{k_{n f}}{k_{f}}=\frac{\left(k_{s}-2 k_{f}\right)-2 \varphi\left(k_{f}-k_{s}\right)}{\left(k_{s}+2 k_{f}\right)+\varphi\left(k_{f}-k_{s}\right)},
\end{aligned}
$$

where $\varphi$ is the nanoparticle volume fraction $(0 \leq$ $\varphi \leq 0.2$ in which $\varphi=0$ corresponds to the regular Newtonian fluid. Also, $\varphi$ beyond 0.2 is not physically realizable due to accumulation) and $\left(\rho c_{p}\right)_{n f}$ is the heat capacity of the nanofluid.

By using the Rosseland approximation [51], the radiative heat flux $q_{r}$ is given by:

$$
q_{r}=-\frac{4 \sigma^{*}}{3 k^{*}} \frac{\partial T^{4}}{\partial y}=-\frac{16 \sigma^{*}}{3 k^{*}} T^{3} \frac{\partial T}{\partial y}
$$

where $\sigma^{*}$ is the Stefan-Botzman constant and $k^{*}$ is absorption coefficient. It should be noted that by using the Rosseland approximation, the present analysis is limited to optically thick fluids.

It is worth mentioning here that in the previous studies on thermal radiation (see [19-22] and various references therein), $T^{4}$ in Eq. (6) was expanded about the ambient temperature $T_{\infty}$. That means to simply replace $T^{3}$ in Eq. (6) with $T_{\infty}^{3}$. Now, Eq. (3) can be expressed as:

$$
\begin{aligned}
u \frac{\partial T}{\partial x} & +w \frac{\partial T}{\partial r}=\left(\alpha_{n f}+\frac{16 \sigma^{*} T_{\infty}^{3}}{3 k^{*}\left(\rho c_{p}\right)_{n f}}\right) \frac{1}{r} \frac{\partial}{\partial r}\left(r \frac{\partial T}{\partial r}\right) \\
& +\frac{1}{\left(\rho c_{p}\right)_{n f}} q^{\prime \prime \prime} .
\end{aligned}
$$

However, if we avoid the above-mentioned assumption, the radiative heat flux in Eq. (3) results in a highly nonlinear radiation expression, which is the subject of the current study. Hence, the energy equation for nonlinear thermal radiation will be:

$$
\begin{aligned}
u \frac{\partial T}{\partial x} & +w \frac{\partial T}{\partial r}=\frac{1}{r} \frac{\partial}{\partial r}\left(\left(\alpha_{n f}+\frac{16 \sigma^{*} T^{3}}{3 k^{*}\left(\rho c_{p}\right)_{n f}}\right) r \frac{\partial T}{\partial r}\right) \\
& +\frac{1}{\left(\rho c_{p}\right)_{n f}} q^{\prime \prime \prime}
\end{aligned}
$$

The term $q^{\prime \prime \prime}$ in the right-hand side of Eq. (3) is due to non-uniform heat source, which is defined [34] as:

$$
q^{\prime \prime \prime}=\frac{k_{n f} U}{x \nu_{f}}\left[A^{*}\left(T_{w}-T_{\infty}\right) e^{-\eta}+B^{*}\left(T-T_{\infty}\right)\right],
$$

where $A^{*}$ and $B^{*}$ are the coefficients of exponentially decaying space and temperature-dependent heat source/sink, respectively. It is to be noted that the case $A^{*}>0, B^{*}>0$ corresponds to internal heat source and $A^{*}<0, B^{*}<0$ corresponds to internal heat sink.

Consider the following similarity transformations [39]:

$$
\begin{aligned}
& \eta=\frac{r^{2}-a^{2}}{2 a} \sqrt{\frac{U}{\nu_{f} x}}, \quad \psi=\sqrt{U \nu_{f} x} a f(\eta), \\
& \frac{T-T_{\infty}}{T_{w}-T_{\infty}}=\theta(\eta)
\end{aligned}
$$

where $\psi$ is the stream function and the velocity components are defined as:

$$
u=\frac{1}{r} \frac{\partial \psi}{\partial r}, \quad w=-\frac{1}{r} \frac{\partial \psi}{\partial x},
$$

which identically satisfy Eq. (1).

Substituting Eqs. (5), (9), and (10) in Eqs. (2) and $(8)$, we obtain a system of dimensionless non-linear ordinary differential equations:

$$
\begin{gathered}
\frac{1}{(1-\varphi)^{2.5}\left[(1-\varphi)+\varphi \frac{\rho_{s}}{\rho_{f}}\right]}\left[(1+2 \gamma \eta) f^{\prime \prime \prime}+2 \gamma f^{\prime \prime}\right] \\
+f f^{\prime \prime}-\left(f^{\prime}\right)^{2}+\frac{(1-\varphi)+\varphi\left(\frac{\rho_{s}}{\rho_{f}}\right)\left(\frac{\beta_{s}}{\beta_{f}}\right)}{(1-\varphi)+\varphi \frac{\rho_{s}}{\rho_{f}}} \lambda_{T} \theta=0
\end{gathered}
$$




$$
\begin{aligned}
& \frac{1}{\operatorname{Pr}} \frac{1}{(1-\varphi)+\varphi \frac{\left(\rho c_{p}\right)_{s}}{\left(\rho c_{p}\right)_{f}}} \\
& \quad\left[\left(\frac{k_{n f}}{k_{f}}+R_{d}\left(1+\left(\theta_{w}-1\right) \theta\right)^{3} \theta^{\prime}(1+2 \gamma \eta)\right)^{\prime}\right. \\
& \left.\quad+\frac{k_{n f}}{k_{f}}\left(A^{*} e^{-\eta}+B^{*} \theta\right)\right]+f \theta^{\prime}-N f^{\prime} \theta=0,
\end{aligned}
$$

subject to boundary conditions:

$$
\begin{aligned}
& f^{\prime}=1+S_{1} f^{\prime \prime}, \quad f=0, \quad \theta=1+S_{2} \theta^{\prime}, \\
& \quad \text { at } \eta=0, \\
& f^{\prime} \rightarrow 0, \quad \theta \rightarrow 0 \quad \text { as } \eta \rightarrow \infty,
\end{aligned}
$$

where $\lambda_{T}$ is the thermal buoyancy parameter $\left(\lambda_{T}=0\right.$ corresponds to the case when there is no thermal buoyancy effect, i.e., forced convection flow situation, while $\lambda_{T}>0$ and $\lambda_{T}<0$ correspond to assisting and opposing flows, respectively), $\gamma$ is the curvature parameter $(\gamma=0$ represents a flat plate, while $\gamma=$ 1 represents vertical cylinder), $R_{d}$ is the radiation parameter, and $S_{1}$ and $S_{2}$ are the velocity and thermal slip parameters, which are defined as:

$$
\begin{array}{rlrl}
\lambda_{T} & =\frac{g \beta_{f}\left(T_{w}-T_{\infty}\right) L^{2}}{U_{0}^{2} x}, & \gamma & =\sqrt{\frac{\nu_{f} L}{U_{0} a^{2}}}, \\
R_{d} & =\frac{16 \sigma^{*} T_{\infty}^{3}}{3 k_{f} k^{*}}, & S_{1}=B_{1} \sqrt{\frac{U_{0}}{\nu_{f} L}}, \\
S_{2} & =K_{1} \sqrt{\frac{U_{0}}{\nu_{f} L}} . &
\end{array}
$$

The parameters of practical interest for the present problem are the skin-friction coefficient and the local Nusselt number, which are respectively given by the following expressions [2,24]:

$$
\begin{aligned}
C_{f} & =\frac{\tau_{w}}{\rho_{f} U_{0}^{2}}, \\
\mathrm{Nu} & =\frac{q_{w} x}{k_{f}\left(T_{w}-T_{\infty}\right)},
\end{aligned}
$$

where $\tau_{w}$ is the shear stress at the surface of the cylinder and $q_{w}$ is the surface heat flux from the surface of the cylinder, which are given by:

$$
\begin{aligned}
& \tau_{w}=-\mu_{n f}\left(\frac{\partial u}{\partial y}\right)_{r=a}, \\
& q_{w}=-k_{n f}\left(\frac{\partial T}{\partial y}\right)_{r=a}+\left(q_{r}\right)_{w} .
\end{aligned}
$$

Using Eq. (15) in Eq. (16), the skin friction and local Nusselt number in dimensionless form are:

$$
\begin{aligned}
& \sqrt{\operatorname{Re}} C_{f}=-\frac{\left.f^{\prime \prime}(0) \bar{x}\right)}{(1-\varphi)^{2.5}}, \\
& \frac{\mathrm{Nu}}{\sqrt{\operatorname{Re}}}=-\left[\frac{k_{n f}}{k_{f}}+R_{d}\left(1+\left(\theta_{w}-1\right) \theta(0)\right)^{3}\right] \theta^{\prime}(0) \bar{x} .
\end{aligned}
$$

\section{Series solution by means of HAM}

For HAM solutions, the appropriate initial guesses can be made as:

$$
\begin{aligned}
& f_{0}(\eta)=\frac{1}{1+S_{1}}[1-\exp (-\eta)], \\
& \theta_{0}(\eta)=\frac{1}{1+S_{2}} \exp (-\eta),
\end{aligned}
$$

and the auxiliary linear operators, $L_{f}(f)$ and $L_{\theta}(\theta)$, which are the linear parts of the respective equations, are:

$$
\begin{aligned}
& L_{f}(f)=\frac{d^{3} f}{d \eta^{3}}-\frac{d f}{d \eta}, \\
& L_{\theta}(\theta)=\frac{d^{2} \theta}{d \eta^{2}}-\theta,
\end{aligned}
$$

satisfying the following properties:

$$
\begin{aligned}
& L_{f}\left(C_{1}+C_{2} \exp (\eta)+C_{3} \exp (-\eta)\right)=0, \\
& L_{\theta}\left(C_{4} \exp (\eta)+C_{5} \exp (-\eta)\right)=0,
\end{aligned}
$$

where $C_{i}, i=1-5$, are constants.

\subsection{Zeroth-order deformation problem}

$$
\begin{aligned}
& (1-p) L_{f}\left[f(\eta ; p)-f_{0}(\eta)\right]=p \hbar_{f} N_{f}[f, \theta], \\
& (1-p) L_{\theta}\left[\theta(\eta ; p)-\theta_{0}(\eta)\right]=p \hbar_{\theta} N_{\theta}[f, \theta]
\end{aligned}
$$

where $p \in[0,1]$ denotes the embedding parameter, $\hbar$ indicates the non-zero auxiliary parameter, and:

$$
\begin{aligned}
N_{f}[f, \theta]= & \frac{1}{(1-\varphi)^{2.5}\left[(1-\varphi)+\varphi \frac{\rho_{s}}{\rho_{f}}\right]} \\
& {\left[(1+2 \gamma \eta) \frac{\partial^{3} f(\eta, p)}{\partial \eta^{3}}+2 \gamma \frac{\partial^{2} f(\eta, p)}{\partial \eta^{2}}\right] } \\
& +f(\eta, p) \frac{\partial^{2} f(\eta, p)}{\partial \eta^{2}}-\left(\frac{\partial f(\eta, p)}{\partial \eta}\right)^{2} \\
& +\frac{(1-\varphi)+\varphi\left(\frac{\rho_{s}}{\rho_{f}}\right)\left(\frac{\beta_{s}}{\beta_{f}}\right)}{(1-\varphi)+\varphi \frac{\rho_{s}}{\rho_{f}}} \lambda_{T} \theta(\eta, p),
\end{aligned}
$$




$$
\begin{aligned}
& N_{\theta}[f, \theta]=\frac{1}{\operatorname{Pr}} \frac{1}{(1-\varphi)+\varphi \frac{\left(\rho c_{p}\right)_{s}}{\left(\rho c_{p}\right)_{f}}} \\
& {\left[\left(\frac{k_{n f}}{k_{f}}+R_{d}\left(1+\left(\theta_{w}-1\right) \theta(\eta, p)\right)^{3} \frac{\partial \theta(\eta, p)}{\partial \eta}(1+2 \gamma \eta)\right)^{\prime}\right.} \\
& \left.+\frac{k_{n f}}{k_{f}}\left(A^{*} e^{-\eta}+B^{*} \theta(\eta, p)\right)\right]+f(\eta, p) \frac{\partial \theta(\eta, p)}{\partial \eta} \\
& -N \frac{\partial f(\eta, p)}{\partial \eta} \theta(\eta, p),
\end{aligned}
$$

subject to the following boundary conditions:

$$
\begin{aligned}
& f(0, p)=0, f^{\prime}(0, p)=1+S_{1} f^{\prime \prime}(0, p), \quad f^{\prime}(\infty, p)=0, \\
& \theta(0, p)=1+S_{2} \theta^{\prime}(0, p), \quad \theta(\infty, p)=0 .
\end{aligned}
$$

\section{2. mth-order deformation problems}

Differentiating the zeroth-order deformation problems (24) for $m$ times with respect to $p$, then dividing it by $m$ ! and setting $p=0$, we get the following higherorder deformation problems:

$$
\begin{aligned}
& L_{f}\left[f_{m}(\eta)-\chi_{m} f_{m-1}(\eta)\right]=\hbar_{f} R_{m}^{f}(\eta), \\
& L_{\theta}\left[\theta_{m}(\eta)-\chi_{m} \theta_{m-1}(\eta)\right]=\hbar_{\theta} R_{m}^{\theta}(\eta) .
\end{aligned}
$$

And the boundary conditions are:

$$
\begin{aligned}
& f_{m}(0)=0, \quad f_{m}^{\prime}(0)=S_{1} f_{m}^{\prime \prime}(0), \quad f_{m}^{\prime}(\infty)=0, \\
& \theta_{m}(0)=S_{2} \theta_{m}^{\prime}(0), \quad \theta_{m}(\infty)=0,
\end{aligned}
$$

where $f_{m}(\eta), \theta_{m}(\eta), R_{m}^{f}(\eta)$ and $R_{m}^{\theta}(\eta)$ are calculated by Eqs. (27) and (28), as shown in Box I, and:

$$
\chi_{m}= \begin{cases}0 & m \leq 1 \\ 1 & m \geq 1\end{cases}
$$

For $p=0$ and $p=1$, we can write:

$$
\begin{array}{ll}
f(\eta, 0)=f_{0}(\eta), & f(\eta, 1)=f(\eta), \\
\theta(\eta, 0)=\theta_{0}(\eta), & \theta(\eta, 1)=\theta(\eta),
\end{array}
$$

and with variation of $p$ from 0 to $1, f(\eta, p)$, and $\theta(\eta, p)$ vary from initial solutions $f_{0}(\eta)$ and $\theta_{0}(\eta)$ to final solutions $f(\eta)$ and $\theta(\eta)$, respectively. By Taylor's series, we have:

$$
\begin{aligned}
& f(\eta, p)=f_{0}(\eta)+\sum_{m=1}^{\infty} f_{m}(\eta) p^{m}, \\
& f_{m}(\eta)=\left.\frac{1}{m !} \frac{\partial^{m} f(\eta, p)}{\partial \eta^{m}}\right|_{p=0}, \\
& \theta(\eta, p)=\theta_{0}(\eta)+\sum_{m=1}^{\infty} \theta_{m}(\eta) p^{m}, \\
& \theta_{m}(\eta)=\left.\frac{1}{m !} \frac{\partial^{m} \theta(\eta, p)}{\partial \eta^{m}}\right|_{p=0} .
\end{aligned}
$$

The value of the auxiliary parameter is chosen in such a way that these two series (33) and (34) are convergent at $p=1$.

Eq. (27) represents the system of nonhomogeneous linear differential equations whose

$$
\begin{aligned}
f_{m}(\eta)= & \frac{1}{m !} \frac{\partial^{m} f(\eta, p)}{\partial \eta^{m}}, \quad \theta_{m}(\eta)=\frac{1}{m !} \frac{\partial^{m} \theta(\eta, p)}{\partial \eta^{m}}, \\
R_{m}^{f}(\eta)= & \frac{1}{(1-\varphi)^{2.5}\left[(1-\varphi)+\varphi \frac{\rho_{s}}{\rho_{f}}\right]\left[(1+2 \gamma \eta) f_{m-1}^{\prime \prime \prime}+2 \gamma f_{m-1}^{\prime \prime}\right]}+\sum_{n=0}^{m-1} f_{m-1-n} f_{n}^{\prime \prime}-\sum_{n=0}^{m-1} f_{m-1-n}^{\prime} f_{n}^{\prime} \\
+ & \frac{(1-\varphi)+\varphi\left(\frac{\rho_{s}}{\rho_{f}}\right)\left(\frac{\beta_{s}}{\beta_{f}}\right)}{(1-\varphi)+\varphi \frac{\rho_{s}}{\rho_{f}}} \lambda_{T} \theta_{m-1}, \\
R_{m}^{\theta}(\eta)= & \frac{1}{\operatorname{Pr}} \frac{1}{(1-\varphi)+\varphi \frac{\left(\rho c_{p}\right)_{s}}{\left(\rho c_{p}\right)_{f}}}\left[\left(\frac{k_{n f}}{k_{f}}+R_{d}\left(1+\left(\theta_{w}-1\right) \theta_{m-1}\right)^{3} \theta_{m-1}^{\prime}(1+2 \gamma \eta)\right)^{\prime}+\frac{k_{n f}}{k_{f}}\left(A^{*} e^{-\eta}+B^{*} \theta_{m-1}\right)\right] \\
& +\sum_{n=0}^{m-1} f_{m-1-n} \theta_{n}^{\prime}-N \sum_{n=0}^{m-1} f_{m-1-n}^{\prime} \theta_{n} .
\end{aligned}
$$


general solutions are the sum of complementary and particular solutions, which can be expressed as:

$$
\begin{aligned}
& f_{m}(\eta)=f_{m}^{*}(\eta)+C_{1}^{m}+C_{2}^{m} e^{\eta}+C_{3}^{m} e^{-\eta}, \\
& \theta_{m}(\eta)=\theta_{m}^{*}(\eta)+C_{4}^{m} e^{\eta}+C_{5}^{m} e^{-\eta},
\end{aligned}
$$

where the constants $C_{i}^{m}(i=1,2, \cdots 5)$ can be calculated through the boundary conditions in Eq. (28).

\subsection{Convergence analysis}

In HAM method, it is essential to ensure the convergence of the series solution. As pointed out by Liao [46], the convergence rate of approximation for the HAM solution strongly depends on the value of the auxiliary parameter, $\hbar$. This auxiliary parameter provides us with great freedom to adjust and control the convergence region of the series solution. Hence, in order to seek the permissible values of $\hbar_{f}$ and $\hbar_{\theta}$, the functions of $f^{\prime \prime}(0)$ and $\theta^{\prime}(0)$ are plotted at the 20th order of approximations. The values of $\hbar_{f}, \hbar_{\theta}$, and $\hbar_{\phi}$ are selected in such a way that curves are parallel to horizontal axis, i.e., $\hbar$-axis [46]. Figures 2 and 3 clearly depict the acceptable range for values of $\hbar_{f}(-0.8 \leq$ $\left.\hbar_{f} \leq-0.1\right)$ and $\hbar_{\theta}\left(-0.6 \leq \hbar_{\theta} \leq-0.1\right)$. The current calculations are based on the value of $\hbar_{f}=\hbar_{\theta}=-0.5$. In order to ensure the convergence of solutions, Table 1 is given. This table clearly shows that convergence is obtained at the 30th order of approximations.

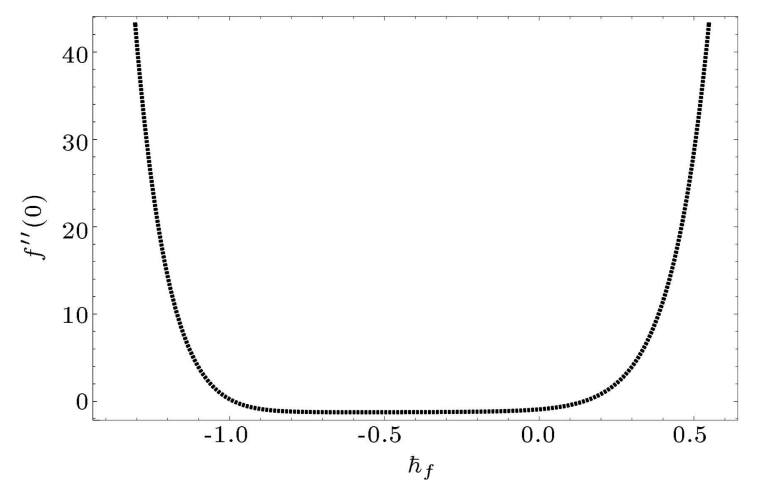

Figure 2. $\hbar$ curve for $f$ at 20 th order of approximation.

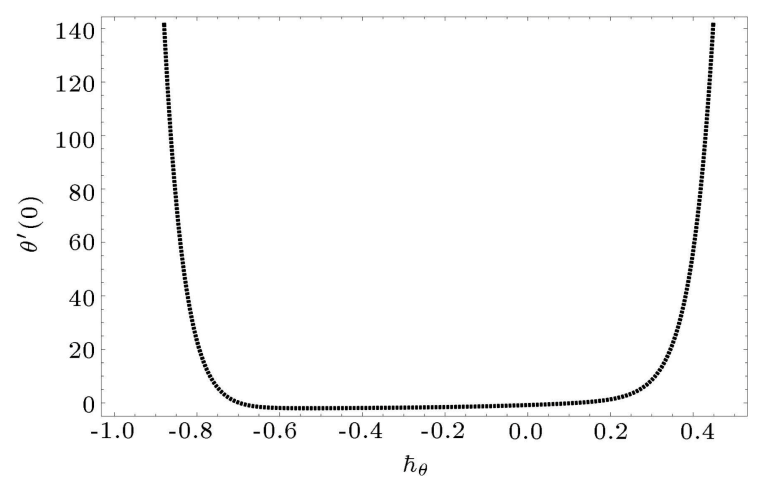

Figure 3. $\hbar$ curve for $\theta$ at 20 th order of approximation.
Table 1. Convergence of HAM solutions for $f^{\prime \prime}(0)$ and $\theta^{\prime}(0)$.

\begin{tabular}{ccc}
\hline $\begin{array}{c}\text { Order of } \\
\text { approximations }\end{array}$ & $-\boldsymbol{f}^{\prime \prime}(\mathbf{0})$ & $-\boldsymbol{\theta}^{\prime}(\mathbf{0})$ \\
\hline 1 & 0.9385 & 0.9879 \\
5 & 0.9806 & 1.4439 \\
10 & 0.9906 & 1.7711 \\
15 & 0.9926 & 1.9382 \\
20 & 0.9925 & 2.0166 \\
25 & 0.9921 & 2.0486 \\
28 & 0.9919 & 2.0555 \\
29 & 0.9919 & 2.0564 \\
30 & 0.9919 & 2.0564 \\
\hline
\end{tabular}

\section{Results and discussion}

To get clear physical insight into the present problem, computations have been carried out using the method described in the previous section for variations in the governing parameters and the results are graphically illustrated in Figures 4-14. In the present study, the following default parameter values are adopted for computations: $\varphi=0.1, \lambda_{T}=0.1, A^{*}=0.01$, $B^{*}=0.01, S_{1}=0.1, S_{2}=0.2, \gamma=1, \theta_{w}=1.5$, $N=1, \operatorname{Pr}=6.2$ (which physically corresponds to water based nanofluid), and $R_{d}=1$. Therefore, all graphs and tables correspond to these values unless otherwise stated.

In the present study, we have used Copper $(\mathrm{Cu})$

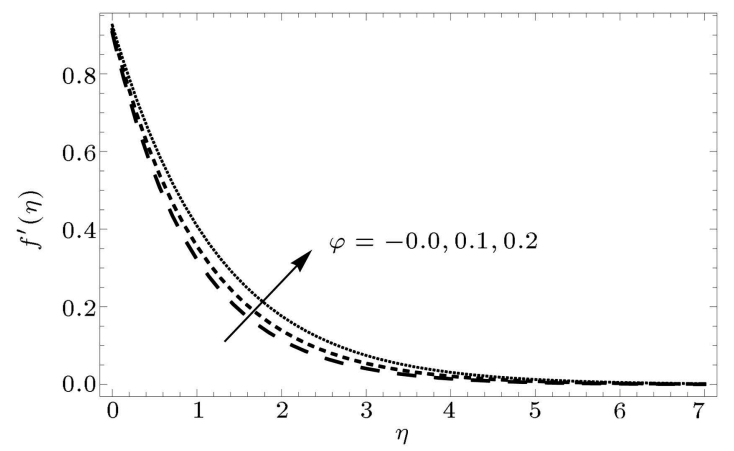

Figure 4. Effect of $\varphi$ on the velocity.

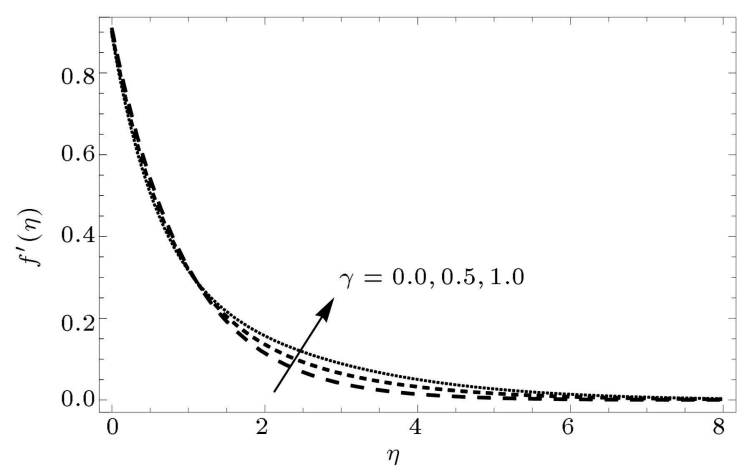

Figure 5. Effect of $\gamma$ on the velocity. 


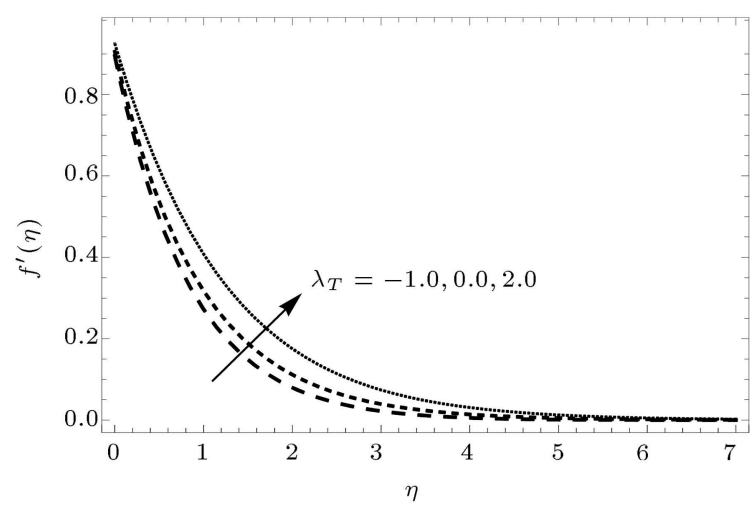

Figure 6. Effect of $\lambda_{T}$ on the velocity.

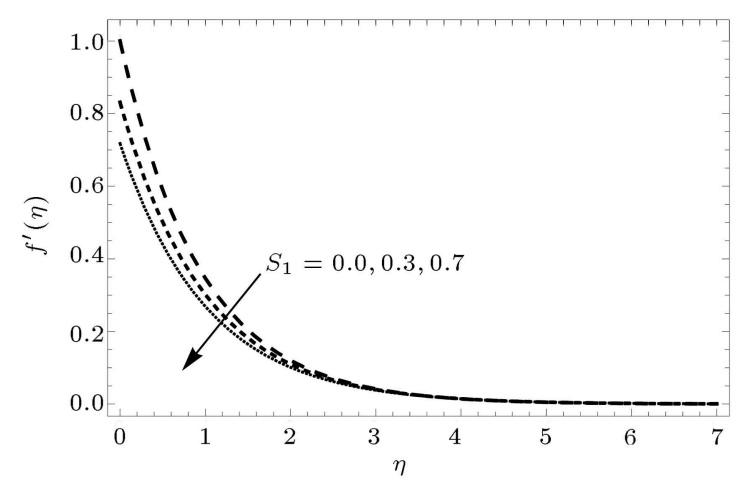

Figure 7. Effect of $S_{1}$ on the velocity.

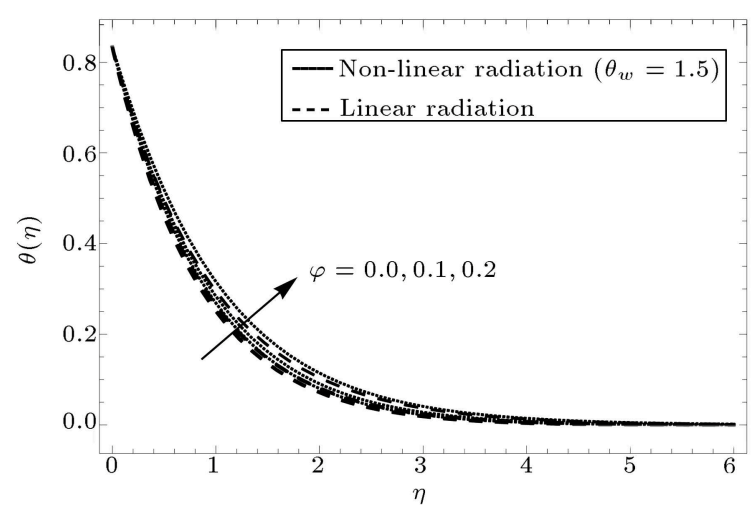

Figure 8. Effect of $\varphi$ on the temperature for $\theta_{w}=1.5$.

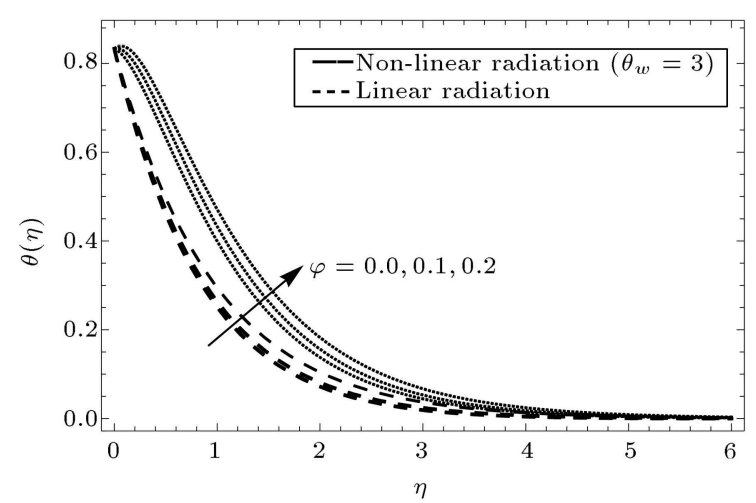

Figure 9. Effect of $\varphi$ on the temperature for $\theta_{w}=3$.

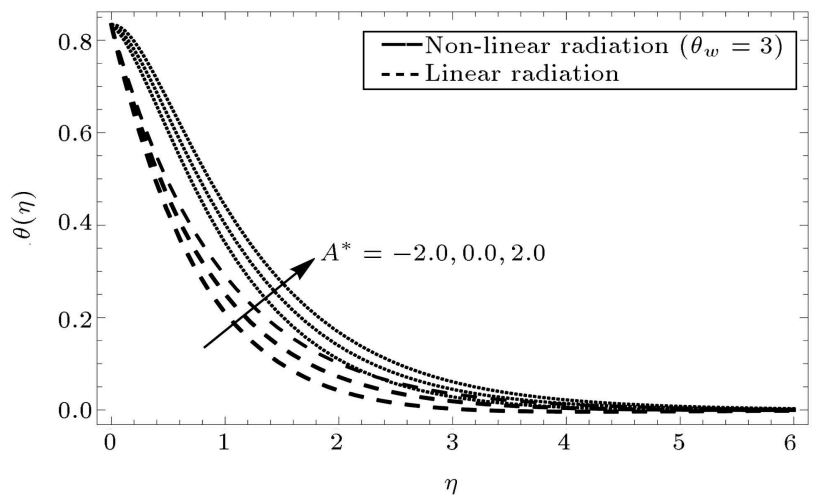

Figure 10. Effect of $A^{*}$ on the temperature.

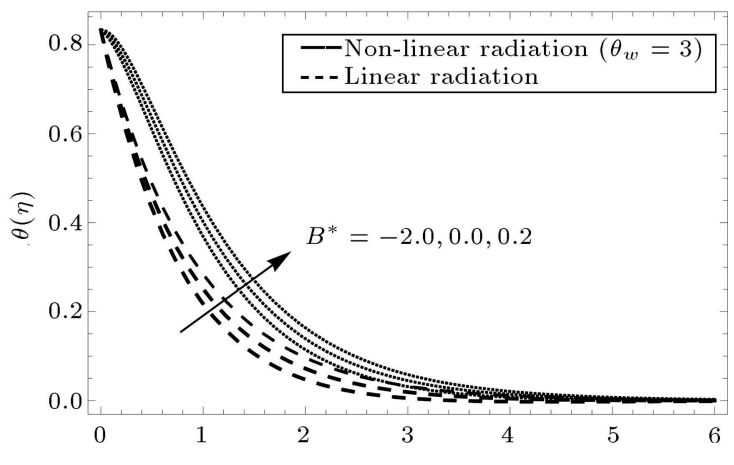

Figure 11. Effect of $B^{*}$ on the temperature.

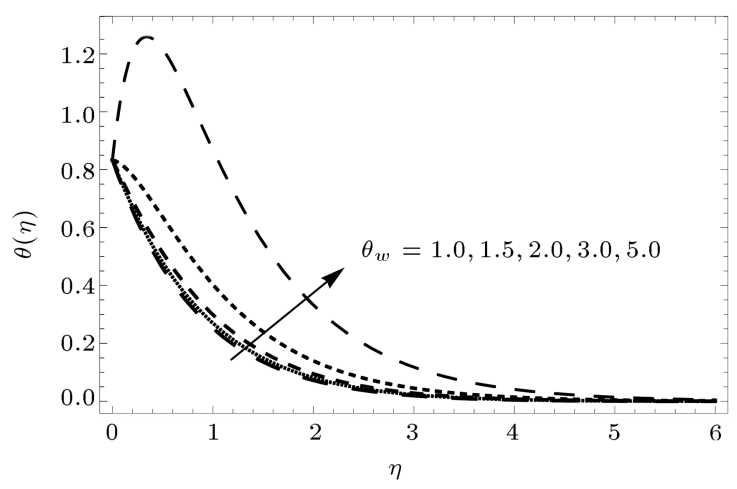

Figure 12. Effect of $\theta_{w}$ on the temperature.

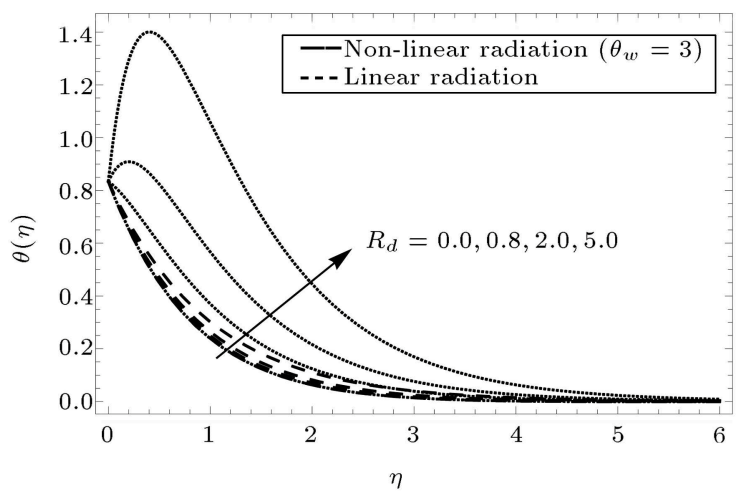

Figure 13. Effect of $R_{d}$ on the temperature. 


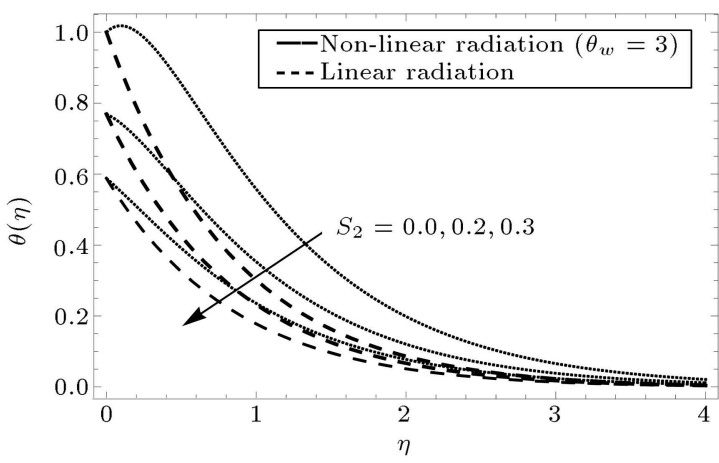

Figure 14. Effect of $S_{2}$ on the temperature.

nanoparticles with water as base fluid and data related to the thermophysical properties of the fluid and nanoparticles are listed in Table 2. For the verification of accuracy of the applied HAM, a comparison of the present results corresponding to the values of $\left[-\theta^{\prime}(0)\right]$ with the available published results of Grubka et al. [52], Ali et al. [53], Abel et al. [54], and Yadav et al. [55] in the case of pure fluid $(\varphi=0)$ is made numerically and presented in Table 3 . The results are found in excellent agreement. Therefore, we are confident that the present results are accurate.

The variation of dimensionless velocity, $f^{\prime}(\eta)$, for different values of nanoparticle volume fraction, $\varphi$, is shown in Figure 4 for $\mathrm{Cu}$-water nanofluid. It can be observed that the dimensionless velocity increases with the nanoparticle volume fraction. This is due to the fact that increase in $\varphi$ leads to increase in energy transportation and, hence, fluid flow. It is also worth noting that velocity of the nanofluid is higher than that of the pure fluid $(\varphi=0)$.

The effect of curvature parameter, $\gamma$, on the veloc- ity within the boundary layer of $\mathrm{Cu}$-water nanofluid is presented in Figure 5. The velocity is found to increase with increase in curvature parameter. This is due to the fact that increase in $\gamma$ leads to shrinking of the cylinder and, hence, the space provided for the free stream of the nanofluid increases. Thus, the nanofluid velocity tends to be free stream velocity. It is important to note in the figure that for a flat plate $(\gamma=0)$, the dimensionless velocity is smaller than that for the vertical cylinder $(\gamma=1)$.

The behavior of thermal buoyancy parameter, $\lambda_{T}$, on the momentum boundary layer can be visualized from Figure 6. The effect of increasing values of the thermal buoyancy parameter, $\lambda_{T}$, is to increase the velocity, $f^{\prime}(\eta)$. Physically, $\lambda_{T}>0$ means heating of the fluid or cooling of the surface (assisting flow), $\lambda_{T}<0$ means cooling of the fluid or heating of the surface (opposing flow), and $\lambda_{T}=0$ means absence of convection currents (forced convection flow). Hence, increase in the value of $\lambda_{T}$ can lead to increase in the temperature difference between fluid and surface. This leads to enhancement of the velocity $f^{\prime}(\eta)$.

Figure 7 demonstrates the effect of velocity slip parameter $\left(S_{1}\right)$ for vertical stretching cylinder on velocity. With increase in $S_{1}$, the velocity is found to decrease in the vicinity of the cylinder surface. This is because with increase in $S_{1}$, the flow velocity near the stretching wall will not be equal to the stretching velocity of the wall. Hence, pulling of the stretching wall can only be partly transmitted to the fluid. Consequently, fluid velocity decreases. $S_{1}=0$ corresponds to the case when there is no velocity slip effect.

Figures 8 and 9 present the influence of nanoparti-

Table 2. Thermophysical properties of fluid and nanoparticles [1].

\begin{tabular}{|c|c|c|c|c|}
\hline $\begin{array}{c}\text { Physical } \\
\text { properties }\end{array}$ & $C_{p}(\mathrm{~J} / \mathrm{kg} \mathrm{K})$ & $\rho\left(\mathrm{kg} / \mathrm{m}^{\mathbf{3}}\right)$ & $K(\mathbf{W} / \mathbf{m} \mathbf{K})$ & $\beta_{X} 10^{-5}(1 / K)$ \\
\hline Water & 4179 & 997.1 & 0.613 & 21 \\
\hline $\mathrm{Cu}$ & 385 & 8933 & 400 & 1.67 \\
\hline
\end{tabular}

Table 3. Comparison of numerical values of $\left[-\theta^{\prime}(0)\right]$ in the present study with previous studies for special cases $(\varphi=0$, $\lambda_{T}=0, A^{*}=0, B^{*}=0, S_{1}=0, S_{2}=0, \gamma=0$, and $\left.\theta_{w}=1\right)$.

\begin{tabular}{ccccccc}
\hline $\mathbf{P r}$ & $\boldsymbol{N}$ & $\begin{array}{c}\text { Grubka et al. } \\
{[\mathbf{5 2}]}\end{array}$ & $\begin{array}{c}\text { Ali et al. } \\
{[\mathbf{5 3}]}\end{array}$ & $\begin{array}{c}\text { Abel et al. } \\
{[\mathbf{5 4}]}\end{array}$ & $\begin{array}{c}\text { Yadav et al. } \\
{[\mathbf{5 5}]}\end{array}$ & $\begin{array}{c}\text { Present study } \\
\text { (by HAM) }\end{array}$ \\
\hline 0.72 & 1 & 0.8086 & 0.8086 & - & 0.8087 & 0.8087 \\
1 & 1 & 1.0000 & 1.0000 & 1.0002 & 1.0000 & 1.0000 \\
3 & -1 & 0.0 & - & - & -0.0039 & 0.0 \\
3 & 0 & 1.1652 & - & - & 1.1545 & 1.1642 \\
3 & 1 & 1.9237 & 1.9237 & 1.9236 & 1.9237 & 1.9216 \\
3 & 2 & 2.5097 & - & - & 2.5159 & 2.5090 \\
10 & 0 & 2.3080 & - & - & 2.3000 & 2.3000 \\
\hline
\end{tabular}


cle volume fraction, $\varphi$, in both cases of linear (obtained from the solution to Eq. (7)) and non-linear (obtained from the solution to Eq. (8)) radiative heat fluxes on the temperature $\theta(\eta)$ when $\theta_{w}=1.5$ and $\theta_{w}=3.0$, respectively. It is clear that as the nanoparticle volume fraction increases, temperature $\theta(\eta)$ increases. This is due to the physical fact that thermal conductivity increases with increase in copper volume fraction and, hence, thermal boundary layer thickness increases. It can also be observed that linear and non-linear results match better when $\theta_{w}$ approaches 1 .

The impact of space-dependent heat source/sink parameter, $A^{*}$, on the dimensionless temperature is depicted in Figure 10 for both linear and non-linear radiation cases. From Figure 10, it is observed that increase in space-dependent heat source/sink parameter leads to increase in the temperature in both linear and non-linear radiation cases. It is due to the fact that when $A^{*}>0$, thermal boundary layer generates energy, resulting in increasing temperature, and energy is absorbed when $A^{*}<0$, resulting in significant temperature drop near the boundary layer. Similar effect is observed in Figure 11 for the temperaturedependent heat source/sink parameter $B^{*}$ in both linear and non-linear radiative heat flux cases.

The effect of temperature ratio parameter, $\theta_{w}$, on the dimensionless temperature has been plotted in Figure 12. It is observed that increase in $\theta_{w}$ leads to intensification of temperature. Increasing value of $\theta_{w}$ indicates larger wall temperature than the ambient temperature. Hence, temperature increases with $\theta_{w}$. We can find an overshoot near the surface of the cylinder for higher values of $\theta_{w}(>5)$.

Figure 13 demonstrates the influence of radiation parameter, $R_{d}$, on the dimensionless temperature, $\theta(\eta)$, in both cases of linear and non-linear radiative heat fluxes. Increase in $R_{d}$ leads to increase in temperature. This is due to the fact that increase in radiation releases the heat energy from the flow, which in turn decreases the thickness of the thermal boundary layer. It can also be observed from the figure that temperature becomes larger with larger values of radiation parameter in the case of non-linear radiation. However, this is not the case for linear radiation. Thus, it can be concluded that non-linear radiation has higher effect on the temperature than the linear radiation.

The impact of thermal slip parameter $S_{2}$ on the dimensionless temperature is depicted in Figure 14 for both linear and non-linear radiation cases. Increase in $S_{2}$ results in decrease in temperature. The main reason is that with increase in thermal slip parameter $S_{2}$, less heat is transferred to the fluid from the stretching wall and, thus, temperature decreases.

The density of nanofluid increases with increase in nanoparticle volume fraction. Due to this, skin friction (the drag force at the surface of the cylinder) also
Table 4. Numerical values of skin friction for various values of $S_{1}, \varphi, \gamma$, and $\lambda_{T}$.

\begin{tabular}{ccccc}
\hline $\boldsymbol{S}_{\boldsymbol{1}}$ & $\boldsymbol{\lambda}_{\boldsymbol{T}}$ & $\boldsymbol{\varphi}$ & $\boldsymbol{\gamma}$ & $(\mathrm{Re})^{\frac{1}{2}} \boldsymbol{C}_{\boldsymbol{f}}\left(\frac{\mathbf{1}}{\overline{\boldsymbol{x}}}\right)$ \\
\hline 0.0 & 0.1 & 0.1 & 1.0 & 1.7172 \\
0.1 & & & & 1.6270 \\
& 0.0 & & & 1.6461 \\
& -0.1 & & & 1.6655 \\
& & 0.2 & & 2.2833 \\
& & 0.0 & & 1.1617 \\
& & 0.0 & 0.8891 \\
\hline
\end{tabular}

increases with increasing nanoparticle volume fraction. This can be observed in Table 4. Moreover, it is noted that skin friction is higher for the nanofluid than for the pure fluid $(\varphi=0)$. It can also be observed in Table 4 that the skin friction is higher for the vertical cylinder $(\gamma=1)$ than for the flat plate $(\gamma=0)$; this is due to large lateral surface area. It can be seen in Table 4 that with increase in thermal buoyancy parameter, $\lambda_{T}$, there is a decrease in skin friction. This agrees with the physical behavior that with increase in thermal buoyancy, velocity increases and, hence, there is decrease in momentum boundary layer and skin friction. On the other hand, the skin friction decreases with slip velocity.

Table 5 provides the numerical values of Nusselt number (rate of heat transfer at the wall) for different values of $\varphi, A^{*}, B^{*}, S_{2}, \gamma$, and $\theta_{w}$ with the other parameters fixed for both linear and non-linear radiation cases. It shows that Nusselt number is an increasing function of $\varphi$, i.e., the Nusselt number is higher for nanofluid $(\varphi=0.1)$ than for pure fluid $(\varphi=0)$. Increase in radiation parameter, $R_{d}$, leads to increase in Nusselt number. This is due to the fact that for large values of radiation parameter, the thermal boundary layer thickens. In the absence of thermal slip, $S_{2}$, the Nusselt number is found to be higher and it decreases with increasing thermal slip. The Nusselt number is a decreasing function of both space-dependent $\left(A^{*}\right)$ and temperature-dependent $\left(B^{*}\right)$ heat source/sink parameters. It is observed from the table that the Nusselt number is higher for a cylinder than for flat plate. The table also depicts that the Nusselt number is lower for linear radiation than for non-linear one.

\section{Concluding remarks}

The present work investigated the influence of nonlinear thermal radiation on mixed convective boundary layer flow of Copper-Water nanofluid over a vertical stretching circular cylinder. The effects of non-uniform heat source and slip flow were also incorporated. 
Table 5. Numerical values of Nusselt number for various values of $\varphi, \gamma, A^{*}, B^{*}, R_{d}$, and $S_{2}$.

\begin{tabular}{|c|c|c|c|c|c|c|c|}
\hline \multirow{2}{*}{$\varphi$} & \multirow{2}{*}{$\gamma$} & \multirow{2}{*}{$\boldsymbol{R}_{d}$} & \multirow{2}{*}{$A^{*}$} & \multirow{2}{*}{$B^{*}$} & \multirow{2}{*}{$S_{2}$} & \multicolumn{2}{|c|}{$\mathrm{Nu}(\mathrm{Re})^{\frac{-1}{2}}\left(\frac{1}{\bar{x}}\right)$} \\
\hline & & & & & & Linear radiation & $\begin{array}{c}\text { Non-linear radiation } \\
\qquad\left(\theta_{w}=1.1\right)\end{array}$ \\
\hline \multirow[t]{10}{*}{0.0} & 0.0 & 1.0 & 0.01 & 0.01 & 0.0 & 3.0203 & 3.4088 \\
\hline & & 2.0 & & & & 4.1351 & 4.7189 \\
\hline & & & 0.02 & & & 3.0158 & 3.4036 \\
\hline & & & & 0.02 & & 3.0139 & 3.4013 \\
\hline & & & & & 0.3 & 2.5085 & 2.5404 \\
\hline & 1.0 & 1.0 & 0.01 & 0.01 & 0.0 & 3.1862 & 3.6379 \\
\hline & & 2.0 & & & & 4.6339 & 5.4299 \\
\hline & & & 0.02 & & & 3.1817 & 3.6327 \\
\hline & & & & 0.02 & & 3.1798 & 3.6304 \\
\hline & & & & & 0.3 & 2.5120 & 2.7042 \\
\hline \multirow[t]{10}{*}{0.1} & 0.0 & 1.0 & 0.01 & 0.01 & 0.0 & 3.4704 & 3.8333 \\
\hline & & 2.0 & & & & 4.5134 & 5.0456 \\
\hline & & & 0.02 & & & 3.4634 & 3.8252 \\
\hline & & & & 0.02 & & 3.4602 & 3.8216 \\
\hline & & & & & 0.3 & 2.6571 & 2.8682 \\
\hline & 1.0 & 1.0 & 0.01 & 0.01 & 0.0 & 3.6785 & 4.1122 \\
\hline & & 2.0 & & & & 5.0903 & 5.8498 \\
\hline & & & 0.02 & & & 3.6714 & 4.1042 \\
\hline & & & & 0.02 & & 3.6682 & 4.1006 \\
\hline & & & & & 0.3 & 2.8172 & 3.0688 \\
\hline
\end{tabular}

Different from the linear radiation, the present problem was governed by an additional temperature ratio parameter. The solutions were computed analytically by Homotopy Analysis Method (HAM). The major conclusions are listed below:

1. Unlike previously reported results on thermal radiation effect, the current results are valid for both small $\left(\theta_{w} \leq 1\right)$ and large temperature differences $\left(\theta_{w}>1\right)$ between surface of the cylinder and environment. The results presented clearly indicate that the temperature ratio parameter has significant effect on heat transfer characteristics;

2. Intensifying slip effect leads to reduction in velocity as well as temperature;

3. The non-linear thermal radiation effect is favorable for the scientific and engineering processes involving high thermal requirements when the temperature is highly enhanced by increasing the values of radiation parameter in the non-linear case;

4. By varying the nanoparticle volume fraction, the flow and heat transfer characteristics could be controlled;

5. Nusselt number decreases with rise in space- or time-dependent heat source/sink parameters. On the other hand, the opposite trend is observed as radiation varies;

6. Both Nusselt number and skin-friction are higher for nanofluid than for pure fluid. Also, they are higher for vertical cylinder than for flat plate.

\section{Nomenclature}

$u, w \quad$ Velocity components in the axial and radial directions $\left(\mathrm{ms}^{-1}\right)$

a Radius of the cylinder (m)

$x \quad$ Axial coordinate of the cylinder (m)

$U_{0} \quad$ Reference velocity $\left(\mathrm{ms}^{-1}\right)$

$L \quad$ Characteristic length (m)

$T_{\infty} \quad$ Ambient temperature (K) 
$T_{0} \quad$ Reference temperature $(\mathrm{K})$

$N \quad$ Temperature exponent

$B_{1} \quad$ Velocity slip parameter

$K_{1} \quad$ Thermal slip parameter

$\beta_{s}, \beta_{f} \quad$ Coefficients of thermal expansion of the solid and fluid fractions $\left(\mathrm{K}^{-1}\right)$

$\rho_{s}, \rho_{f} \quad$ Densities of the solid and fluid fractions $\left(\mathrm{kg} \mathrm{m}^{-3}\right)$

$q_{r} \quad$ Radiative heat flux $\left(\mathrm{Wm}^{-2}\right)$

$k_{n f} \quad$ Thermal conductivity of the nanofluid $\left(\mathrm{Wm}^{-1} \mathrm{~K}^{-1}\right)$

$k_{f}, k_{s} \quad$ Thermal conductivity of the fluid and the solid fractions $\left(\mathrm{Wm}^{-1} \mathrm{~K}^{-1}\right.$ )

$f \quad$ Function related to the velocity field

$\theta$ Dimensionless temperature in the nanofluid,

$U \quad$ Stretching velocity $\left(\mathrm{ms}^{-1}\right), U=U_{0} x / L$

$T_{w}(x) \quad$ Prescribed surface temperature $(\mathrm{K})$, $T_{w}(x)=T_{\infty}+T_{0}(x / L)^{N}$

$\bar{x} \quad$ Dimensionless axial coordinate, $\bar{x}=x / L$

Re Local Reynolds number, $\operatorname{Re}=U_{0} L / \nu_{f}$

$\operatorname{Pr} \quad$ Prandtl number, $\operatorname{Pr}=\nu_{f}\left(\rho c_{p}\right)_{f} / k_{f}$

$\theta_{w} \quad$ Temperature ratio parameter, $\theta_{w}=T_{w} / T_{\infty}$

\section{Subscripts}

$f \quad$ Base fluid

$s \quad$ Nanoparticles

$n f \quad$ Nanofluid

\section{Superscript}

Primes denote differentiation with respect to $\eta$

\section{References}

1. Choi, S.U.S. "Enhancing thermal conductivity of fluids with nanoparticles", ASME, USA, pp. 99-105, FED 231/MD (1995).

2. Dinarvand, S., Abbassi, A., Hosseini, R., and Pop, I. "Homotopy analysis method for mixed convective boundary layer flow of a nanofluid over a vertical circular cylinder", Thermal Science, 19, pp. 549-561 (2015).

3. Oztop, H.F. and Abu-Nada, E. "Numerical study of natural convection in partially heated rectangular enclosures filled with nanofluids", Int. J. Heat Fluid Flow, 29, pp. 1326-1336 (2008).

4. Hayat, T., Ijaz Khan, M., Farooq, M., Alsaedi, A., and Imran Khan, M. "Thermally stratified stretching flow with Cattaneo-Christov heat flux", Int. J. Heat and Mass Transfer, 106, pp. 289-294 (2017).
5. Hayat, T., Ijaz Khan, M., Farooq, M., Yasmeen T., and Alsaedi, A. "Water-carbon nanofluid flow with variable heat flux by a thin needle", J. Molecular Liquids, 224, pp. 786-791 (2016).

6. Hayat, T., Tamoor, M., Khan, M.I., and Alsaedi, A. "Numerical simulation for nonlinear radiative flow by convective cylinder", Results in Physics, 6, pp. 10311035 (2016).

7. Hayat, T., Khan, M.I., Waqas, M., and Alsaedi, A "Newtonian heating effect in nanofluid flow by a permeable cylinder", Results in Physics, 7, pp. 256262 (2017).

8. Hayat, T., Waqas, M., Ijaz Khan, M., and Alsaedi, A. "Analysis of thixotropic nanomaterial in a doubly stratified medium considering magnetic field effects", Int. J. Heat and Mass Transfer, 102, pp. 1123-1129 (2016).

9. Hayat, T., Khan, M.I., Waqas, M., Yasmeen, T., and Alsaedi, A. "Viscous dissipation effect in flow of magneto nanofluid with variable properties", $J$. Molecular Liquids, 222, pp. 47-54 (2016).

10. Alsaedi, A., Ijaz Khan, M., Farooq, M., Gull, N., and Hayat, T. "Magnetohydrodynamic (MHD) stratified bioconvective flow of nanofluid due to gyrotactic microorganisms", Advanced Powder Technology, 28, pp. 288-298 (2017).

11. Hashim and Khan, M. "A revised model to analyze the heat and mass transfer mechanisms in the flow of Carreau nanofluids", Int. J. Heat Mass Transf., 103, pp. 291-297 (2016).

12. Hayat, T., Qayyum, S., Waqas, M., and Alsaedi, A. "Thermally radiative stagnation point flow of Maxwell nanofluid due to unsteady convectively heated stretched surface", .J Mol. Liq., 224, pp. 801-810 (2016).

13. Malik, M.Y., Imad Khan, Hussain, A. and Salahuddin, T. "Mixed convection flow of MHD EyringPowell nanofluid over a stretching sheet: A numerical study", AIP Advances, 5, 117118 (2016). DOI:10.1063/1.4935639.

14. Hussain, A., Malik, M.Y., Salahuddin, T., Bilal, S., and Awais, M. "Combined effects of viscous dissipation and Joule heating on MHD Sisko nanofluid flow over stretching cylinder", Journal of Molecular Liquids, 231, pp. 341-352 (2017).

15. Prasad, K.V., Vajravelu, K., Vaidya, H., and Santhi, S.R. "Axisymmetric flow of a nanofluid past a vertical slender cylinder in the presence of a transverse magnetic field", J. Nanofluids, 5, pp. 101-109 (2016).

16. Prasad, K.V., Vajravelu, K., Vaidya, H., and Datti, P.S. "Axisymmetric flow over a vertical slender cylinder in the presence of chemically reactive species", J. Appl. Comput. Math. (2015). DOI: 10.1007/s40819015-0121-z

17. Prasad, K.V., Vajravelu, K., and Vaidya, H. "MHD Casson nanofluid flow and heat transfer at a stretching sheet with variable thickness", J. Nanofluids, 5, pp. 423-435 (2016). 
18. Prasad, K.V., Vaidya, H., Vajravelu, K., Datti, V., and Umesh, V. "Axisymmetric mixed convective MHD flow over a slender cylinder in the presence of chemically reaction", Int. J. App. Mech. and Eng., 21, pp. 121141 (2016).

19. Pal, D., Mandal, G., and Vajravelu, K. "MHD convection-dissipation heat transfer over a non-linear stretching and shrinking sheets in nanofluids with thermal radiation", Int. J. Heat Mass Transf., 65, pp. 481-490 (2013).

20. Zheng, L., Zhang, C., Zhang, X., and Zhang, J. "Flow and radiation heat transfer of a nanofluid over a stretching sheet with velocity slip and temperature jump in porous medium", J. Franklin Inst., 350, pp. 990-1007 (2013).

21. Lin, Y., Zheng, L., and Zhang, X. "Radiation effects on Marangoni convection flow and heat transfer in pseudo-plastic non-Newtonian nanofluids with variable thermal conductivity", Int. J. Heat Mass Transf., 77, pp. 708-716 (2014).

22. Zhang, C., Zheng, L., Zhang, X., and Chen, G. "MHD flow and radiation heat transfer of nanofluids in porous media with variable surface heat flux and chemical reaction", Appl. Math. Model., 39, pp. 165-181 (2015).

23. Magyari, E. and Pantokratoras, A. "Note on the effect of thermal radiation in the linearized Rosseland approximation on the heat transfer characteristics of various boundary layer flows", Int. Comm. in Heat and Mass Transfer, 38, pp. 554-556 (2011).

24. Khan, M., Malik, R., and Hussain, M. "Nonlinear radiative heat transfer to stagnation-point flow of Sisko fluid past a stretching cylinder", AIP Advances, 6, 055315 (2016). DOI: 10.1063/1.4950946.

25. Hayat, T., Ijaz Khan, M., Waqas, M., Alsaedi, A., and Farooq, M. "Numerical simulation for melting heat transfer and radiation effects in stagnation point flow of carbon-water nanofluid", Computer Methods in Applied Mechanics and Engineering, 315, pp. 10111024 (2017).

26. Hayat, T., Ijaz Khan, M., Farooq, M., Alsaedi, A., and Yasmeen, T. "Impact of Marangoni convection in the flow of carbon-water nanofluid with thermal radiation", Int. J. of Heat and Mass Transfer, 106, pp. 810-815 (2017).

27. Yasmeen, T., Hayat, T., Ijaz Khan, M., Imtiaz, M., and Alsaedi, A. "Ferrofluid flow by a stretched surface in the presence of magnetic dipole and homogeneousheterogeneous reactions", Journal of Molecular Liquids, 223, pp. 1000-1005 (2016).

28. Hayat, T., Ijaz Khan, M., Imtiaz, M., and Alsaedi, A. "Similarity transformation approach for ferromagnetic mixed convection flow in the presence of chemically reactive magnetic dipole", AIP Physics of Fluids, 28 (2016). DOI: $10.1063 / 1.4964684$.

29. Farooq, M., Ijaz Khan, M., Waqas, M., Hayat, T., and Alsaedi, A. "MHD stagnation point flow of viscoelastic nanofluid with non-linear radiation effects", J. Molecular Liquids, 221, pp. 1097-1103 (2016).
30. Khan, M.I., Kiyani, M.Z., Malik, M.Y., Yasmeen, T., Khan, M.W.A., and Abbas, T. "Numerical investigation of magnetohydrodynamic stagnation point flow with variable properties", Alexandria Engineering Journal, 55, pp. 2367-2373 (2016).

31. Khan, M., Ali, H., and Hussain, M. "Magnetohydrodynamic flow of Carreau fluid over a convectively heated surface in the presence of non-linear radiation", $J$. Mag. Mag. Mat., 412, pp. 63-68 (2016).

32. Hayat, T., Qayyum, S., Alsaedi, A., and Waqas, M. "Simultaneous influences of mixed convection and nonlinear thermal radiation in stagnation point flow of Oldroyd-B fluid towards an unsteady convectively heated stretched surface", J. Mol. Liq., 224, pp. 811817 (2016).

33. Mustafa, M., Mushtaq, A., Hayat, T., and Ahmad, B. "Nonlinear radiation heat transfer effects in the natural convective boundary layer flow of nanofluid past a vertical plate: A numerical study", PLOS ONE, 9(9): e103946 (2014). https://doi.org/10.1371/journal.pone.0103946

34. Hayat, T., Qayyum, S., Alsaedi, A., and Asghar, S. "Radiation effects on the mixed convection flow induced by an inclined stretching cylinder with non-uniform heat source/sink", PLOS ONE, 12(4): e0175584 (2017).

https://doi.org/10.1371/journal.pone.0175584

35. Manjunatha, P.T., Gireesha, B.J., and Prasanna Kumar, B.C. "Thermal analysis of conducting dusty fluid flow in a porous medium over a stretching cylinder in the presence of non-uniform source/sink", Int. J. Mech. Mat. Eng. (2014). DOI: 10.1186/s40712-0140013-8

36. Subhas Abela, M., Jagadish, T., and Mahantesh Nandeppanavar, M. "Effect of non-uniform heat source on MHD heat transfer in a liquid film over an unsteady stretching sheet", Int. J. Non-Linear Mechanics, 44, pp. 990-998 (2009).

37. Muthtamilselvan, M., Prakash, D., and Doh, D.H. "Effect of non-uniform heat generation on unsteady MHD non-Darcian flow over a vertical stretching surface with variable properties", J. Applied Fluid Mechanics, 7, pp. $425-434$ (2014).

38. Hayat, T., Farooq, M., and Alsaedi, A. "Stagnation point flow of carbon nanotubes over stretching cylinder with slip conditions", Open Phys., 13, pp. 188-197 (2015).

39. Hayat, T., Qayyum, A., and Alsaedi, A. "Effects of heat and mass transfer in flow along a vertical stretching cylinder with slip conditions", Eur. Phys. J. Plus, 129(63) (2014). DOI:10.1140/epjp/i2014-140639.

40. Mukhopadhyay, S. "Chemically reactive solute transfer in a boundary layer slip flow along a stretching cylinder", Front Chem. Sci. Eng., 5, pp. 385-391(2011).

41. Sravanthi, C.S. "Homotopy analysis solution of MHD slip flow past an exponentially stretching inclined sheet 
with Soret-Dofour effects", J. the Nigerian Mathematical Society, 35, pp. 208-226 (2016).

42. Masood Khan and Ali, H. "Effects of multiple slip on flow of magneto-carreau fluid along wedge with chemically reactive species", Neural Comput Applic, (2016). DOI: $10.1007 / \mathrm{s} 00521-016-2825-2016$

43. Salahuddin, T., Malik, M.Y., Arif Hussain, Awais, M., and Bilal, S. "Mixed convection boundary layer flow of Williamson fluid with slip conditions over a stretching cylinder by using Keller box method", Int. J. Nonlinear Sciences and Numerical Simulation, 18, pp. 9-17 (2017).

44. Bilal, S., Khalil Ur Rehman, Hamayun Jamil, Malik, M.Y. and Salahuddin, T. "Dissipative slip flow along heat and mass transfer over a vertically rotating cone by way of chemical reaction with Dufour and Soret effects", AIP Advances, 6, 125125 (2016). DOI: http://dx.doi.org/10.1063/1.4973307.

45. Liao, S.J., Beyond Perturbation: Introduction to Homotopy Analysis Method, Boca Raton: Chapman \& Hall/CRC Press (2003).

46. Liao, S.J., Homotopy Analysis Method in Nonlinear Differential Equations, Beijing and Heidelberg: Higher Education Press \& Springer (2012).

47. Hayat, T., Khan, M.I., Waqas, M., Alsaedi, A., and Yasmeen, T. "Diffusion of chemically reactive species in third grade fluid flow over an exponentially stretching sheet considering magnetic field effects", Chinese Journal of Chemical Engineering, 25, pp. 257263 (2017).

48. Hayat, T., Khan, M.I., Alsaedi, A., and Khan, M.I. "Homogeneous-heterogeneous reactions and melting heat transfer effects in the MHD flow by a stretching surface with variable thickness", J. Mol. Liq., 223, pp. 960-968 (2016).

49. Hayat, T., Khan, M.I., Farooq, M., Gull, N., and Alsaedi, A. "Unsteady three-dimensional mixed convection flow with variable viscosity and thermal conductivity", J. Mol. Liq., 223, pp. 297-310 (2016).
50. Hayat, T., Khan, M.I., Farooq, M., Alsaedi, A., and Khan, M.I. "Thermally stratified stretching flow with Cattaneo-Christov heat flux", Int. J. Heat and Mass Transfer, 106, pp. 289-294 (2017).

51. Rosseland, S., Astrophysik und Atomtheoretische Grundlagen, Springer, Berlin, pp. 41-44 (1931).

52. Grubka, L.J. and Bobba, K.M. "Heat transfer characteristics of a continuous stretching surface with variable temperature", J. Heat Transfer, 107, pp. 248250 (1985).

53. Ali, F.M., Nazar, R., Arifin, N.M., and Pop, I. "Effect of Hall current on MHD mixed convection boundary layer flow over a stretched vertical flat plate", Meccanica, 46, pp. 1103-1112 (2011).

54. Abel, M.S., Tawade, J.N., and Nandeppanavar, M.M. "MHD flow and heat transfer for the upper-convected Maxwell fluid over a stretching sheet", Meccanica, 47, pp. 385-393 (2012).

55. Yadav, R.S. and Sharma, P.R. "Effects of porous medium on MHD fluid flow along a stretching cylinder", Annals of Pure and Applied Mathematics, 6, pp. 104-113 (2014).

\section{Biography}

C.S. Sravanthi has been working as an Assistant Professor of Mathematics in the Department of Mathematics at the Maharaja Sayajirao University of Baroda since 2013. In 2008, she obtained MSc degree from S. V. University, Tirupati, India. In 2015, she obtained PhD degree from Mahila University, India. Her main works in mathematics are in the field of fluid mechanics. Since 2008, she has given lectures on mathematics to graduate and post-graduate students of science and engineering in various parts of India. She has given many presentations of papers in international and national conferences. She has published more than 10 papers in the fields of mathematics and fluid mechanics in reputed international journals. 